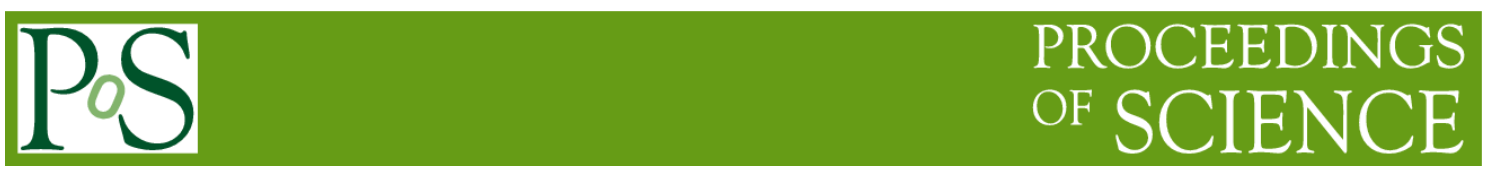

\title{
NOvA latest results
}

\section{Filip Jediný* \\ on behalf of the NOvA Collaboration}

Czech Technical University in Prague

Brehova 7, Prague, Czech Republic

E-mail: filip.jediny@fjfi.cvut.cz

The NOvA experiment is a long-baseline accelerator-based neutrino oscillation experiment. It uses the upgraded NuMI beam from Fermilab and measures electron-neutrino appearance and muon-neutrino disappearance between its onsite near detector and the far detector in Ash River, Minnesota. The beam recently reached the $700 \mathrm{~kW}$ power design benchmark. Goals of the experiment include measurements of theta13, mass hierarchy, theta23 octant and the $C P$ violating phase. This work is a proceeding to a talk which described the experiment and the status of its measurements and which presented the latest results based on the exposure equivalent to $6.05 \times 10^{20} \mathrm{POT}$.

The 15th International Conference on Flavor Physics \& CP Violation 5-9 June, 2017

Prague, Czech Republic

\footnotetext{
${ }^{*}$ Speaker
} 


\section{Introduction}

Neutrino physics, with many interesting topics and questions reaching beyond Standard Model, is being more and more appealing for particle physicists. Despite the huge progress made in recent years, there are still many open questions, regarding i.e. the mass ordering, the mixing angles and the $\mathrm{CP}$ violation in neutrino sector.

The NuMI Off-Axis $v_{\mathrm{e}}$ Appearance experiment (NOvA) was designed to answer some of these questions [1]. Fully operating since 2015, its one of the world's leading neutrino experiments and the flagship of the Intensity Frontier particle physics program of Fermi National Laboratory in Batavia, IL (USA).

NOvA is a long-baseline, two-detector neutrino oscillation experiment looking mostly for appearance of $v_{\mathrm{e}}$ in well-defined NuMI beam of $v_{\mu}$. The beam was recently upgraded so it can be delivering $700 \mathrm{~kW}$ of beam power, with optional change between neutrino and antineutrino mode. From these measurements, NOvA is capable of extracting oscillation parameters: $\Delta m^{2}{ }_{23}$, mixing angle $\theta_{23}, \mathrm{CP}$ violating phase $\delta_{C P}$ and the neutrino mass hierarchy.

\section{NOvA experiment}

To carry out a measurement of sufficient number of oscillated accelerator neutrinos, a so-called 2nd generation experiment has to have a very intensive beam of neutrinos and a massive detector several hundreds of kilometers away. NOvA detects the NuMI beam (same as MINOS [2]) with Far Detector (FD) $810 \mathrm{~km}$ from the target and Near Detector (ND) $1 \mathrm{~km}$ from the target.

\subsection{NuMI Beam}

The source of neutrinos to detect in NOvA detectors is the NuMI beam Neutrinos at the Main Injector. The neutrinos are created by extracting $120 \mathrm{GeV}$ protons from the Main Injector facility at Fermilab, and colliding them with a graphite target, which results in production of secondary mesons $\left(\pi^{+}\right.$particles can decay into $\mu^{+}$and $\left.v_{\mu}\right)$. To meet the physics goals of NOvA, the NuMI beam was upgraded from $300 \mathrm{~kW}$ to $700 \mathrm{~kW}$ of nominal beam power. This is achieved by reducing the $10 \mu$ s-pulse cycle time of the Main Injector via slip-stacking in the recycler ring; increasing the intensity per cycle with 12 Booster batches instead of 11 by installing new RF stations and a new injection kicker magnet; and upgrading the target and horns to accommodate the increased proton intensity.

As a result, we have a $10 \mu$ s beam spill every $1.33 \mathrm{~s}$ in an intensity of $4.9 \times 10^{13}$ protons per pulse (corresponding to $6.0 \times 10^{20}$ protons on target per year of running). From pion decay kinematics, we know the neutrino energy will depend on the characteristic decay angle between the neutrino and the parent pion in the laboratory frame. For $14 \mathrm{mrad}$ off-axis, most pion decays result in neutrinos with $\mathrm{E}=2 \mathrm{GeV}$, some with energy smearing around that value. Therefore, the NOvA detectors placed $14 \mathrm{mrad}$ off the NuMI beam axis will measure a narrow band beam peaked very near the $v_{\mu}$ to $v_{e}$ oscillation maximum at $810 \mathrm{~km}(\mathrm{E} \sim 1.6 \mathrm{GeV})$. The narrow beam energy spectrum strongly reduces the background from feed-down of higher energy neutral-current (NC) neutrino events, the dominant background for neutrino oscillation searches with the detectors placed onaxis.

The oscillation results presented here come from an exposure equivalent to $6.05 \times 1020$ protons on-target in the "full" $14 \mathrm{kton}$ detector. This exposure is more than double that used in the previous publications $[3,4]$. 


\subsection{NOvA Detectors}

The NOvA detectors are finely segmented, $65 \%$ active tracking calorimeters. The segmentation and the overall mechanical structure of the detectors are provided by a lattice of extruded PVC cells with cross sectional size $6 \mathrm{~cm} \times 4 \mathrm{~cm}$. Each cell extends the full width or height of the detector (15.6 $\mathrm{m}$ in the FD, $4.1 \mathrm{~m}$ in the $\mathrm{ND}$ ) and is filled with mineral oil mixed with liquid scintillator (pseudocumene). Secondary particles from neutrino interactions excite the scintillator and the light produced by the scintillator is collected and transported to the end of the cell by a wavelength-shifting ber that terminates on a pixel of a 32-channel avalanche photodiode (APD). The APDs are cooled to $-15{ }^{\circ} \mathrm{C}$ and readout continuously. Each of the 928 layers of the $14 \mathrm{kton}$ FD has 384 cells, for 344000 total channels of readout. The 300ton ND has 206 layers, each with 96 cells plus a muon range stack at the downstream end, made by interleaving steel plates with standard detector layers, totalling 18000 channels.

\section{$2.3 \mathrm{CVN}$}

We omit the description of a very sophisticated simulation and reconstruction chain in NOvA software here. However, the PID of the $v_{e}$ appearance analysis used a very novel convolutional neural network technique [5], and the $v_{\mu}-v_{e}$ joint analysis publication is the first case of a HEP result obtained through the deep learning network, called Convolutional Visual Network (CVN). With minimal event reconstruction, it is possible to build and train a single algorithm which achieved excellent separation of signal and background for both appearance and disappearance oscillation channels.

For the NOvA analysis, a new charged-current (CC) classifier was developed to select a signal sample with improved purity and efficiency. The CVN is a convolutional neural network and was designed using deep learning techniques from the field of computer vision. Recorded hits in the detectors are formed into clusters by grouping hits in time and space to isolate individual interactions. The CVN classifier takes the hits from these clusters, without any further reconstruction, as input and applies a series of trained linear operations to extract complex, abstract classifying features from the image. A multilayer perceptron at the end of the network uses these features to create the classifier output. Training of the CVN was conducted using a set of simulated FD events. Convolutional filters are used to automatically extract features from the raw hit map in both $\mathrm{x}$ and $\mathrm{y}$ views, where the signal hits are treated as pixels in a visual input to the network, which takes advantage of the recent development in GPU computation. When the output of the CVN is used to classify the event, the statistical power of the neural net is equivalent to $30 \%$ more exposure than previous conventional PID techniques, LID and LEM [3,4].

The CVN algorithm is a powerful approach to the problem of event classification and represents a novel proof of concept that CNNs can work extremely well with artificial images like the readout of a sampling calorimeter, or, expectably, of a wide range of detector technologies and analyses. Other possible expansions for CVN in NOvA would include neutral current events, charged current $v_{\tau}$ interactions, dark matter searches or other exotic physics. Initial results have already shown improvements in the efficiency and purity in particle identification over other likelihood methods employed by NOvA and will aid in the future cross-section analyses.

\section{Experimental results}

For the second analysis dataset of $6.05 \times 10^{20} \mathrm{POT}$, two papers were recently published for the $v_{\mu}$ disappearance analysis [6] and the joint appearance and disappearance fit [7]. The $v_{e}$ appearance 
second analysis results are expected to be published later in 2017.

\section{$3.1 v_{\mu}$ disappearance}

A blind analysis, where energy, muon classifier values, and the number of FD beam events were obscured until the analysis was finalized, was performed on the second analysis dataset (more than double of data from previous publications). After unblinding, $78 v_{\mu}$-CC candidate events were observed in the Far Detector with an expected background of $3.4 \mathrm{NC}, 0.23 v_{e}$-CC, $0.27 v_{\tau^{-}}$ $\mathrm{CC}$ events, and 2.7 cosmic-ray-induced events. In the case of no oscillations, $473 \pm 30$ events are predicted. At the best-fit parameters, 82.4 events are expected. Figure 1 shows the measured energy spectrum along with the best fit prediction, with the ratio to the prediction in the absence of oscillations shown in the lower part. The data are fit for oscillations using 19 energy bins of $0.25 \mathrm{GeV}$ width between $0.25-5.0 \mathrm{GeV}$.

The best fit to the second analysis data gives $\Delta m^{2}{ }_{23}=(+2.67 \pm 0.11) \times 10^{-3} \mathrm{eV}^{2}$ and $\sin ^{2} \theta_{23}$ at two degenerate values 0.404 and 0.624 both at the C.L. of $68 \%$ in the normal hierarchy (NH). For the inverted hierarchy, $\Delta m^{2}{ }_{23}=(-2.72 \pm 0.11) \times 10^{-3} \mathrm{eV}^{2}$ and $\sin ^{2} \theta_{23}=0.398$ or 0.618 at $68 \%$ C.L. The best fit has a $\chi^{2} /$ d.o.f. $=41.6 / 17$, which arises mainly from bins in the tail of the energy spectrum that contain little information about the three-flavour oscillations. Restricting the fit to energies below $2.5 \mathrm{GeV}$ reduces the $\chi^{2} /$ d.o.f. to $3.2 / 7$ and does not significantly change the fit results.

Maximal mixing, where $\sin ^{2} \theta_{23}=0.5$, is disfavoured by the data at $2.6 \sigma$ level. Fixing $\sin ^{2} \theta_{23}=0.5$ gives a best fit of $\Delta m^{2}{ }_{23}=2.48 \times 10^{-3} \mathrm{eV}^{2}(\mathrm{NH})$ with a prediction of 77.7 events. Figure 1 shows the difference between the energy spectra for the maximal mixing prediction, in dashed green, and the best fit to our data, in red, for which the mixing is nonmaximal. The $1-2 \mathrm{GeV}$ region is where the oscillation maximum occurs and the events in that range provide the most information about the mixing angle. Visual scanning of the events in this region along with studies of their geometric location and kinematic variables gave results consistent with expectations.

Figure 2 shows $90 \%$ C.L. allowed regions of $\Delta m_{23}^{2}$ and $\sin ^{2} \theta_{23}$ where two separated islands occur, one for each $\theta_{23}$ octant. These new results are consistent with those in our previous publication [3]. Contours from MINOS [2] and T2K [8] are also shown in Figure 2 for comparison.

\subsection{Joint Fit}

The PID of the $v_{e}$ channel was obtained using the CVN technique described in Section 2.3. After the event selection criteria and analysis procedures, the FD data revealed $33 v_{e}$ candidates, of which $8.2 \pm 0.8$ (syst.) events are predicted background. Figure 3 shows the regions of $\sin ^{2} \theta_{23}$ and $\delta_{C P}$ space allowed at three different confidence levels. The likelihood surface is profiled over the parameters $\Delta m^{2}{ }_{23}$ and $\theta_{23}$ while the so called solar parameters $\Delta m^{2}{ }_{2 l}$ and $\theta_{13}$ are fixed.

The significances are calculated using the unified Feldman-Cousins approach to account for the statistical effects of low event count and physical boundaries 9. Combined with the latest NOvA $v_{\mu}$ disappearance data and external constraints from reactor experiments on $\sin ^{2} \theta_{13}$, the hypothesis of inverted mass hierarchy with $\theta_{23}$ in the lower octant is disfavoured at greater than 93\% C.L. 


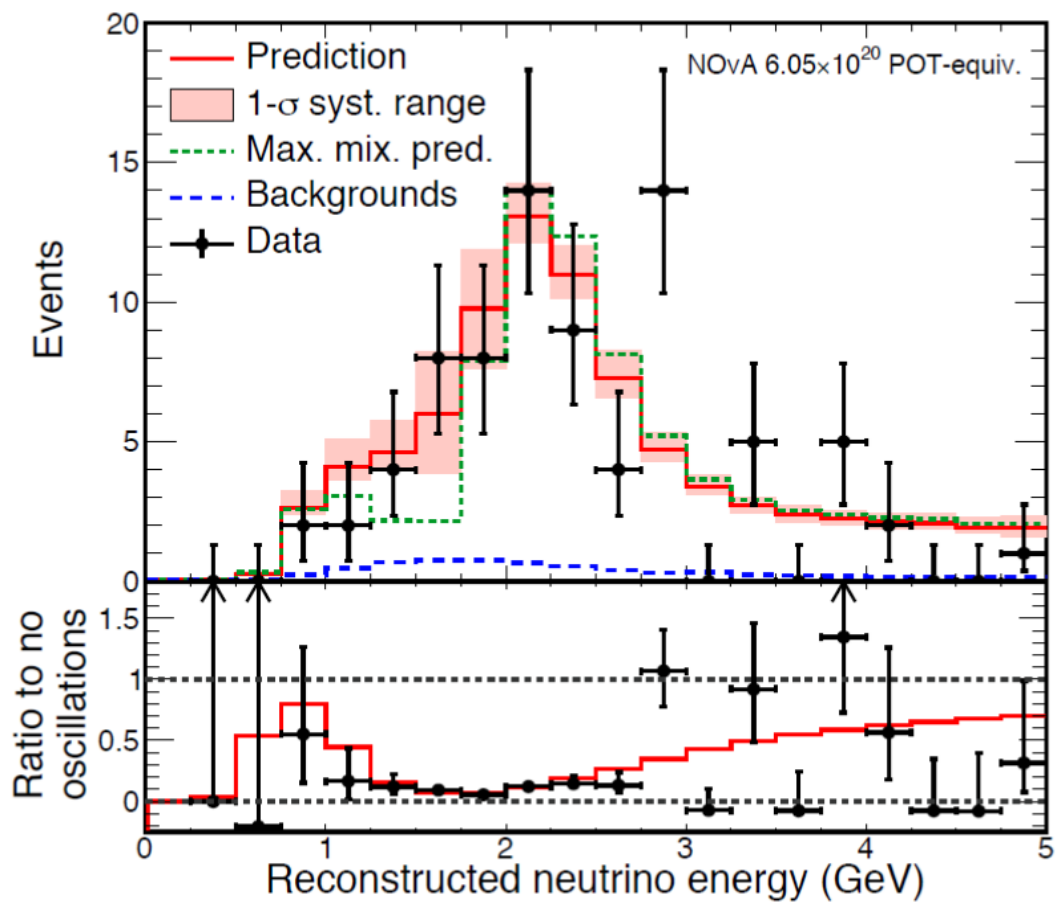

Figure 1: Top: Comparison of the reconstructed energy spectrum of the FD data (black dots) and best-fit prediction (red). The systematic uncertainty band is shaded red. Combined beam and cosmic backgrounds are shown by the dashed blue histogram. The prediction assuming maximal mixing is shown in dashed green. Bottom: The ratio to no-oscillations for data and Monte Carlo after background subtraction.

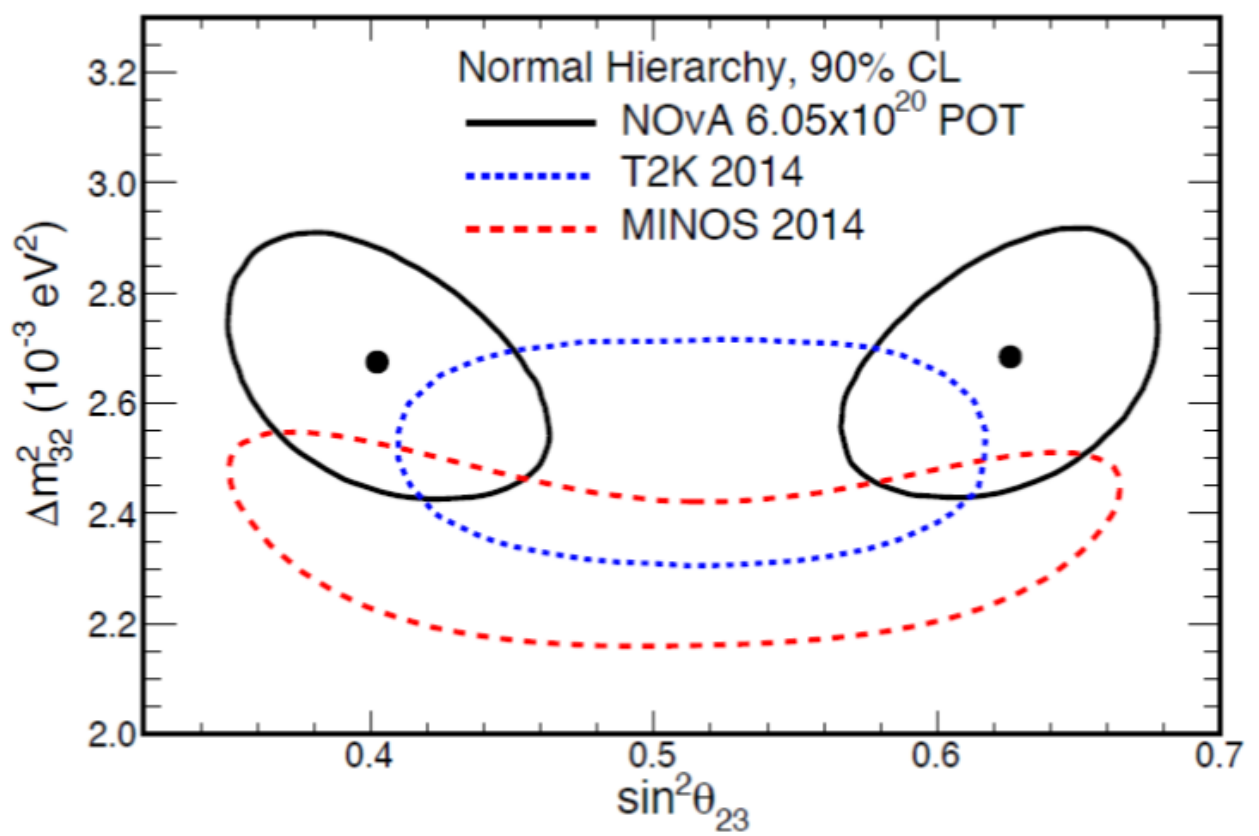

Figure 2: Best fit (black dots) and allowed 90\% C.L. regions (solid black curves) of $\Delta m^{2}{ }_{23}$ and $\sin ^{2} \theta_{23}$ for the Normal Hierarchy. The dashed curves show MINOS [2] and T2K [8] 90\% C.L. contours. 

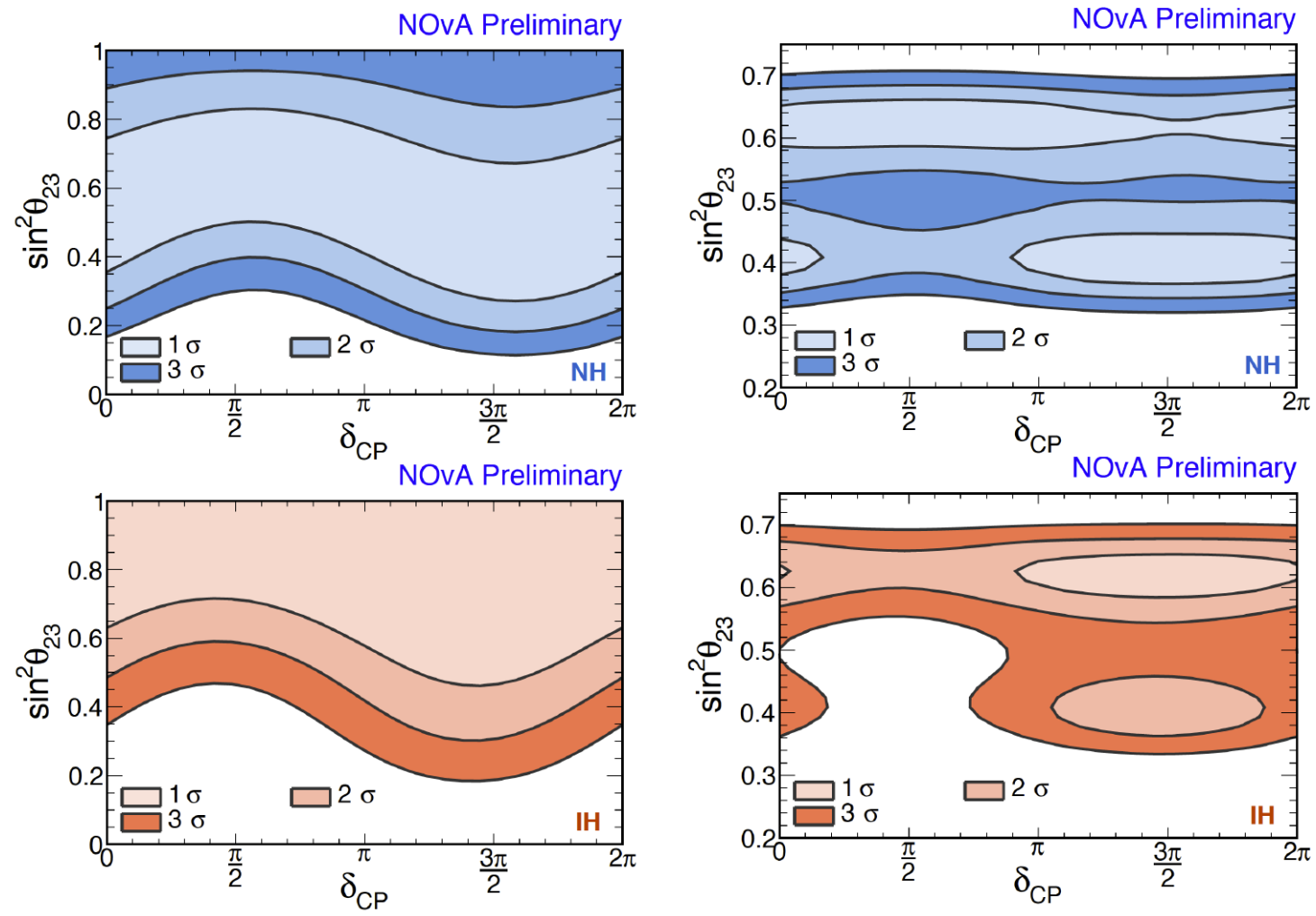

Figure 3: Allowed regions of $\sin ^{2} \theta_{23}$ vs. $C P$ phase $\delta_{C P}$ at 1,2, and $3 \sigma$ : (left) results from $v_{e}$ appearance data and (right) results from the combination of $v_{e}$ appearance and $v_{\mu}$ disappearance data.

\section{Summary}

At NuMI beam intensity of $\sim 6 \times 10^{20}$ POT per year, the expected production rate is estimated to $O\left(10^{14}\right) \mathrm{LDM}$ particle candidates per year, leaving the target area boosted forward into few degrees around the beam direction.

\section{Acknowledgments}

This work was supported by the US Department of Energy; the US National Science Foundation; the Department of Science and Technology, India; the European Research Council; the MSMT CR, Czech Republic; the RAS, RMES, and RFBR, Russia; CNPq and FAPEG, Brazil; and the State and University of Minnesota. We are grateful for the contributions of the staff at the University of Minnesota module assembly facility and Ash River Laboratory, Argonne National Laboratory, and Fermilab. Fermilab is operated by Fermi Research Alliance, LLC under Contract No. De-AC02-07CH11359 with the US DOE.

\section{References}

[1] D. S. Ayres et al. NOvA, FERMILAB-DESIGN-2007-01 (2007).

[2] P. Adamson et al., MINOS Phys. Rev. Lett. 107, 181802 (2011), arXiv:1108.0015v1 [hep-ex].

[3] P. Adamson et al. (NOvA Collaboration), Phys. Rev. D 93, no. 5, 051104 (2016).

[4] P. Adamson et al. (NOvA Collaboration), Phys. Rev. Lett. 116, no. 15, 151806 (2016).

[5] A. Aurisano et al., A Convolutional Neural Network Neutrino Event Classifier (2016) arXiv:1604.01444 [hep-ex]. 
[6] P. Adamson et al. (NOvA Collaboration), Phys. Rev. Lett. 118, 151802 (2017).

[7] P. Adamson et al. (NOvA Collaboration), Constraints on oscillation parameters from nue appearance and numu disappearance in NOvA (2017) arXiv:1703.03328 [hep-ex].

[8] K. Abe et al., Phys. Rev. D 91, 072010 (2015).

[9] G. J. Feldman and R. D. Cousins, Phys. Rev. D 57, 3873 (1998). 